Apidologie, 1984, 15 (2), 99-122

\title{
VERGESELLSCHAFTUNG UND VERSTÄNDIGUNG IM TIERREICH FRAGEN AN DIE SOZIOBIOLOGIE *
}

\author{
ASSOCIATION ET COMMUNICATION DANS LE REGNE ANIMAL \\ QUESTIONS DE SOCIOBIOLOGIE \\ ASSOCIATION AND COMMUNICATION IN ANIMAL KINGDOM \\ QUESTIONS TO SOCIOBIOLOGY
}

Martin LINDAUER

Universität Würzburg, Zoologisches Institut II

Röntgenring 10

D - 87 Würzburg (B.R.D.)

Wir kennen heute weit über 1 Million verschiedene Tierarten, die unseren Erdball bevölkern. Darunter sind ca. 30000 Einzeller, 750000 Arten von Insekten, 20000 Fischarten, 8600 Vögel und nur 3700 Säugetierarten. Diese Vielfalt der Tierwelt ist das Ergebnis eines Grundgesetzes der Evolution, alle nur möglichen ökologischen Nischen als Lebensräume zu besiedeln und in einer Lebensgemeinschaft mit anderen Arten zu nutzen.

Es gibt wohl keine einzige Tierart, die das ganze Leben hindurch ein Einsiedlerdasein führen würde.

Die Soziobiologie beschäftigt sich mit dem Phänomen der Vergesellschaftung der Artgenossen, also mit der Gemeinschaft der Geschlechtspartner, eines Familienverbandes, einer Sippe, insbesondere mit den hochorganisierten Staatengemeinschaften der Termiten, Ameisen und Bienen. Unsere erste Frage ist die nach der Funktion solcher sozialer Verbände.

Zum ersten garantiert die Vereinigung von Männchen und Weibchen die Fortpflanzung. Fortpflanzung meint aber nicht nur Zeugung von Nachkommen ; dafür könnten auch die Weibchen allein sorgen; so pflegen es in der Tat die Wasserflöhe (Daphnia) ; in Abhänigkeit von bestimmten Umweltfaktoren - Jahreszeit, Temperatur, Nahrungsangebot usw. - werden entweder parthenogenetisch «Subitaneier » erzeugt, aus denen wieder parthenogenetisch sich fortpflanzende Weibchen entstehen, oder es werden plötzlich Eier erzeugt, aus denen sich Männchen entwickeln ; gleichzeitig treten dann Weibchen auf, die der Befruchtung

* Herrn F. RutrNer in dankbarer Erinnerung an viele Jahre freundschaftlicher Zusammenarbeit. 
bedürfen, um ihre «Dauereier» zur Entwicklung zu bringen. Das ist eine wichtige Massnahme, weil nur Dauereier schlechte Zeiten, z.B. das Austrocknen einen Tümpels überdauern können.

Die Reblaus wäre niemals zur Plage geworden, würde sie nicht in ihrer komplizierten Fortpflanzungsreihe zwischendurch immer wieder parthenogenetische Generationen einschieben, die die Nachkommenzahl um ein Tausendfaches multiplizieren. Wozu also wird im Normalfall doch immer wieder das männliche Geschlecht eingeschaltet? Die Antwort ist einfach : es wird durch die Kopulation wertvolles Erbgut eingebracht, das mit der Befruchtung der Eizelle eine geradezu grenzenlose neue Kombination der Erbanlagen ermöglicht und damit eine Individualität der Lebewesen garantiert, des weiteren eine Anpassung an die wechselnden Umweltbedingungen erheblich verbessert.

Man sollte auch nicht übersehen, dass die Männchen - gemeinsam mit den Weibchen - noch eine weitere Aufgabe zu erfüllen haben : die Sorge um die Nachkommen bei der Brutpflege, bei der Verteidigung der Jungen gegen fremde Eindringlinge und Räuber.

Wenn sich viele Individuen zu einem Verband zusammenschliessen, wie bei den Sippen der Säugetiere oder bei den hochorganisierten Insektenstaaten, dann ergibt sich die Möglichkeit Spezialisten heranzubilden und durch Arbeitsteilung das Zusammenleben zugunsten einer vermehrten Nachkommenschaft effektiv zu machen. Wenn eine Bienenkönigin im Lauf ihres 5-jährigen Lebens mehr als 1/4 Million Nachkommen zeugt, eine Termitenkönigin ein Vielfaches davon, dann ist dies eben nur im Rahmen eines hochorganisierten Staatengebildes, in dem das Vollweibchen durch die Spezialisten der Arbeiterkasten entlastet wird, möglich.

Die genannten drei Funktionen und Aufgaben haben zur Voraussetzung, dass sich die Partner untereinander verständigen, wie sie zu einem sinnvollen, gemeinsamen Handeln kommen. Ich will die Art und Weise dieser Verständigung in vier Thesen darlegen und in Verbindung damit einige grundlegende Fragen an die Soziobiologie stellen.

\section{THESE 1}

«Jede Verständigung unter Tieren beginnt mit dem Erkennen des Artgenossen $\gg$. Es ist Grundlage für die sexuelle Isolation der Geschlechtspartner und stellt damit die Weichen zur Bildung neuer Arten. Wir fragen, welche Signale dieser intraspezifischen Kommunikation zugrunde liegen. Die Nachtschmetterlinge, die es besonders schwer haben, weil sie weder optische noch akustische Signale benutzen können, sollen uns in die Problematik einführen :

Die Männchen werden durch Duftstoffe, die die Weibchen aus Drüsen an der Abdomenspitze abgeben, aus weiter Entfernung angelockt. Man hat in der Schweiz ein Weibchen des chinesischen Seidenspinners auf einem Balkon in einem Draht- 
käfig ausgesetzt und von den umliegenden Bahnstationen die zugehörigen Männchen freigelassen. Bis auf $4 \mathrm{~km}$ Entfernung kamen sie - bei völliger Dunkelheit angeflogen, um mit dem Weibchen zu balzen. Der Biochemiker ist erstaunt, wie einfach gebaut diese hochwirksamen Sexuallockstoffe sind (Abb. 1). Sie gehen offensichtlich auf einen ungesättigten Alkohol als Stammform zurück, wobei bereits

sexua 1 - Iockstoffe

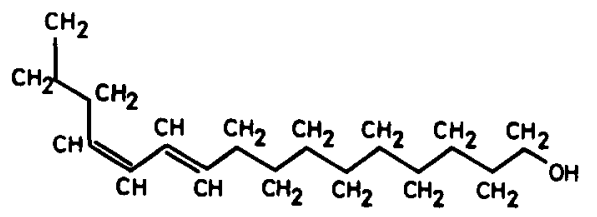

Bombyx mori

trans-10, cis-12-Hexadecadien-1-01 (Bombjko1)

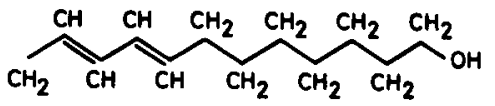

Carpocapsa pomonella

trans-8, trans-10-Dodecadien-1-01

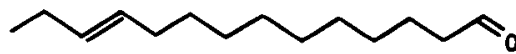

Choristoneura fumiferana

trans-11-Tetradecenal

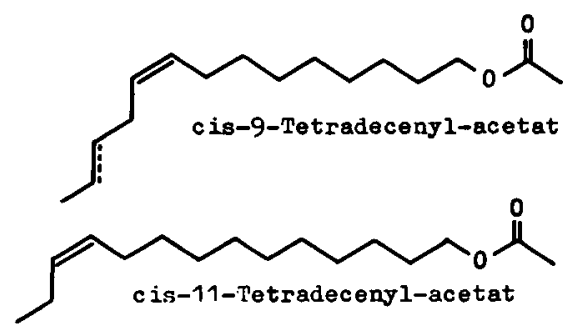

Adoxophyes fasciata

Adoxophyes orana

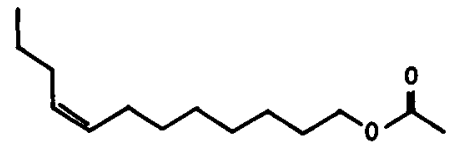

Grapholitha molesta

c is-8-Dodecenjl-ace tat

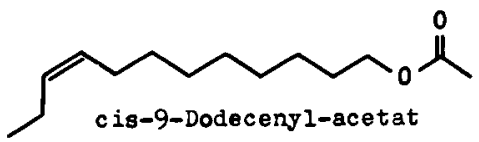

Paralobesia viteana

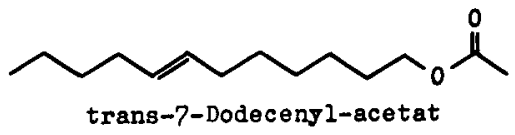

Argyroploce leucotreta

Aвв. 1. - Sexualstoffe einiger Arten von Insektenweibchen 
durch eine einfache Transfiguration ein neues digitales Artsignal geschaffen wird. Dieses wird noch eindeutiger, wenn man den Lockstoff nicht in einer einzigen Komponente, sondern als Mischduft anbietet. Als routinierte Chemiker nutzen diese Nachtfalter sogar das unterschiedliche Mengenverhältnis der einzelnen Duftkomponenten als Artsignal : Die gefürchteten Wicklerarten Adoxophyes orana und $A$. spectrana besitzen die gleichen Isomeren cis-9- und cis-11- Tetradecenyl-acetat.

Erst die richtige proportionale Zusammensetzung weist die Artzugehörigkeit aus. An den Soziobiologen muss aber die Gegenfrage gestellt werden : wenn durch einfache Mutation sowohl eine Konfiguration des Stammoleküls wie auch die quantitative Mischung der einzelnen Komponenten verändert werden, ist damit auch die sexuelle Isolation bei der Balz gesichert, d.h. sind damit schon die Weichen für eine neue Art gestellt?

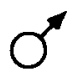

Sphinx ligustri Hyloicus kalmiae Smerinthus ocellata planus

Calasymbolus excaec. Herse convolvuli Psilogramma increta Protoparce sexta Panogena jasmini Theretra japonica " nessus

Amorpha populi Mimas tilice Darapsa myron Rhagastis mongoliana Daphnis nerii Celerio euphorbiae " vespertitio - galii

Pergesa elpenor porcellus

Ampelophaga rubig. Hemaris fuciformis Cephonodes hylas
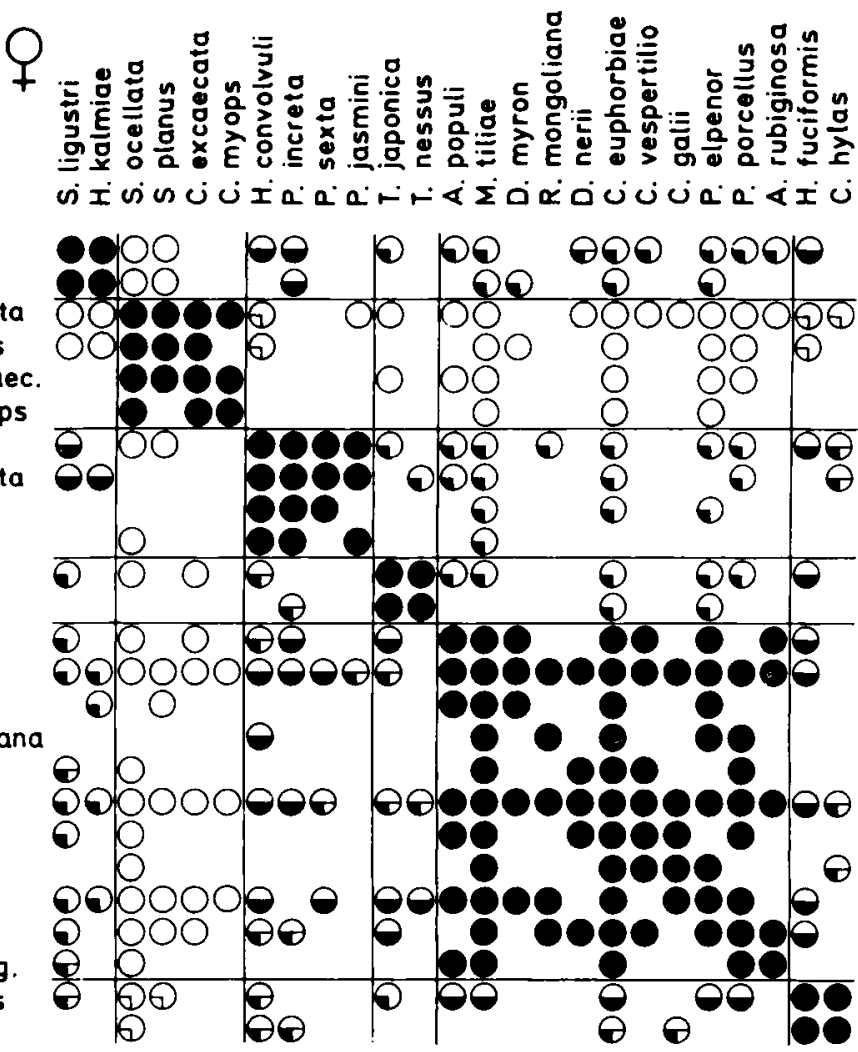

Авв. 2. - Im sog. «Kreuztest》 werden weibliche Sexuallockstoffe

in ihrer Lockwirkung gegenüber Männchen der gleichen bzw. verwandter Arten getestet.

Die Lockstoffe sind nicht streng artspezifisch, lassen aber in ihrer Wirkung dic nähere Verwandtschaft erkennen (aus PRIESNer, 1973).

- Volle Lockstoffunktion.

Keine Wirkung. 
Sicher die Weichen sind gestellt, aber es müssen noch weitere sexuelle Isolationsmechanismen hinzukommen : man zählt ca. 100000 Schmetterlingsarten, die sich über die genannten Sexuallockstoffe verständigen. Die Anzahl der Duftkomponenten, die die Evolution bisher anzubieten hat, geht aber nicht über 2000 hinaus. Dementsprechend ist im sog. Kreuztest, in dem ein in der Natur artspezifischer Sexuallockstoff den Männchen verschiedener Arten angeboten wird, die sexuelle Isolation verwischt (Abb. 2). Nahe verwandte Arten vermögen sich gegenseitig anzulocken (PrIESNer, 1972). In freier Natur wird jedoch die Isolation durch zwei zusätzliche Mechanismen garantiert :

1. durch zeitliche Abgrenzung der Balzaktivität ;

2. durch geographische Barrieren der Population.

Hier sei eine Randbemerkung erlaubt : das Gebiet der Sexualpheromone war zunächst als reine, wertefreie Grundlagenforschung konzipiert. In jüngster Zeit fanden die gewonnenen Erkenntnisse zunehmendes Interesse in der biologischen Schädlingsbekämpfung. Mit Duftstoffen, die den synthetisch hergestellten Sexuallockstoff enthalten, fängt man massenweise die Männchen (einige Gramm/ha reichen hierzu völlig aus).

Mit der sog. Verwirrungsmethode, wobei der Lockstoff in einem Biotop gleichmässig verteilt wird, erzielt man eine Desorientierung der Männchen ; mit Hilfe von Antipheromonen schreckt man sie ab. Beim Baumwollkapselwurm (Pectinophora gossypiala), beim Apfelwickler und bei einigen Vorratsschädlingen sind die Erfolge vielversprechend, beim Schwammspinner gab es einen Fehlschlag : wenn bloss $5 \%$ der Männchen übrig blieben, war im nächsten Jahr wieder ein Massenbefall $\mathrm{zu}$ verzeichnen.

Trotzdem : der Ansatzpunkt ist gut; denn

1. die Sexualpheromone lassen sich leicht synthetisch herstellen;

2. sie sind streng artspezifisch (evtl. nützliche, verwandte Arten bleiben ungeschädigt - im Gegensatz zu Insektiziden);

3. da sie in geringster Menge wirksam sind, bleiben keine Rückstände ;

4. Resistenzbildung oder Immunisierung ist nicht zu befürchten.

Die Variabilität und Vielfalt der Isolationsmechanismen fasziniert den Zoologen. Der Vollständigkeit halber muss ich abschliessend zu unserer 1. These noch von einem Fall «entarteter»Verständigung (im wörtlichen Sinne) berichten : in den Galeriewäldern Südamerikas leben verschiedene Arten von Leuchtkäfern der Gattung Photinus, die nachts durch die Morsezeichen ihrer Männchen einen faszinierenden Feuerzauber bieten. Die flügellosen Weibchen sitzen unten am Boden und antworten jeweils auf das Locksignal ihrer Männchen mit einem artspezifischen Blinkzeichen; daraufhin lassen sich die Männchen zu Boden fallen, um die Kopulation durchzuführen (Abb. 3). 


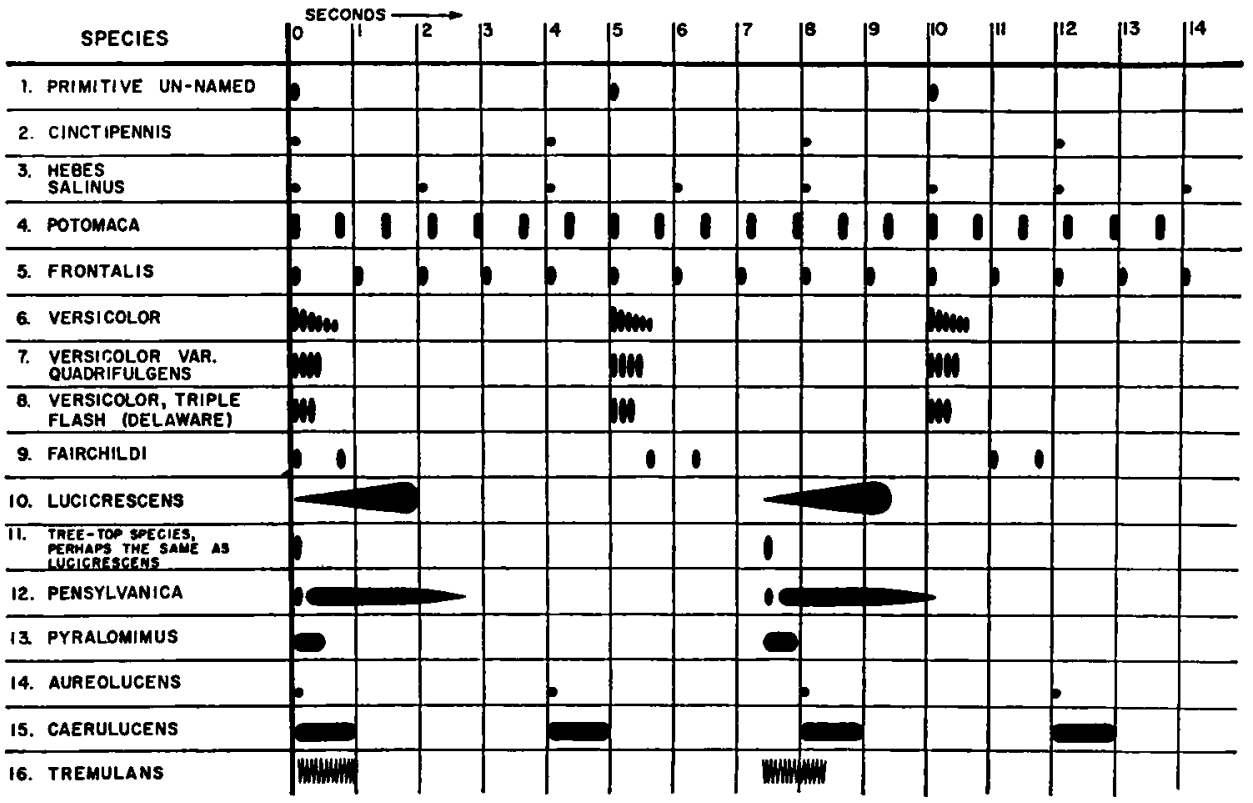

Aвв. 3. - Die Männchen der Gattung Photinus balzen mit artspezifischen Blinkzeichen

So weit, so gut! Neuerdings hat man jedoch entdeckt, dass die Weibchen einer nah verwandten Gattung (Photuris) hinterhältigerweise mit dem artspezifischen Signal auf die Morsezeichen der Photinus-Männchen antworten. Die ahnungslosen Photinus-Männchen lassen sich anlocken, stürzen zu Boden - und werden von den Photuris-Weibchen aufgefressen. Der Biologe hat auch hinter diesem vermeintlich grausamen Spiel einen Sinn gefunden : die Photinus-Männchen besitzen in der Blutlymphe einen Bitterstoff (Lucibufagin, ein Steroid), der sie gegen Räuberfrass schützt. Photuris hat diesen Bitterstoff nicht. Die Mahlzeit von zwei PhotinusMännchen genügt, um die Photuris-Weibchen ebenfalls immun zu machen. Der biologische Sinn ist klar : wo es darum geht, den kostbaren Weibchen von Photuris einen Schutz zu geben, um damit ihre Art zu erhalten, kann man ohne ernste Einbusse auf zwei Männchen einer anderen Art verzichten, da diese sowieso immer in der Überzahl vorhanden sind.

An den Evolutionstheoretiker und Soziobiologen sei aber in diesem Zusammenhang noch eine weitere Frage erlaubt : wieso muss dieser Schutzmechanismus ausgerechnet über den komplizierten Weg der Kommunikation erfolgen ? Warum hat sich Photinus nicht selbst durch entsprechende Mutation einen Bitterstoff in die Blutlymphe eingelagert ? Offenbar sind alle Vorgänge, die mit dem Stoffwechsel zusammenhängen, starrer im genetischen Code verankert als Verhaltenskomponenten und Kommunikationssysteme, die ja durch Lernen zusätzlich modifiziert werden können. 


\section{THESE 2}

Damit sind wir bei unserer zweiten These : «Signalsender und Signalempfänger müssen komplementär und ökonomisch einander zugeordnet sein ». Dies gilt sowohl für den peripheren Empfangsapparat, aber auch für die zentrale Codierung digitaler und analoger Signale. Die Frage erhebt sich, wie einigen sich Sender und Empfänger, welchen Code die einzelnen Signale tragen ? Vereinfacht wird diese Frage durch die Tatsache, dass der Nachrichtenkanal, d.h. jener Weg auf dem die Information übertragen wird, einen Schmalbandfilter darstellt, so dass er an seinem Eingang wie an seinem Ausgang einem Optimalempfänger gleichkommt. Nach diesem Prinzip arbeitet ja auch unsere moderne Nachrichtentechnik. Hierfür einige Beispiele :

Wir finden auf der Antenne der Schmetterlingsmännchen Sensoren, die als «Detektoren» für den Sexuallockstoff der Weibchen spezialisiert sind; bei Drohnen erzeugt nur der Lockstoff der Königin, die 9-cis-12-Transdecensäure, mit wenigen Molekülen Nervenimpulse; die menschliche Nase ist für diesen Duftstoff auch in hoher Konzentration völlig unempfindlich. Die MückenMännchen, die ihre Weibchen am Flugton erkennen, hören nur im Frequenzbereich $600 \mathrm{~Hz}$, was genau dem Flugton der Weibchen entspricht; für ihren eigenen Flugton $(800 \mathrm{~Hz})$ sind sie taub. Bei der Gelbbauchunke hat man im Hörzentrum des Weibchens Neuronenpopulationen gefunden, die ein schmalbandiges Empfindlichkeitsmaximum bei $500 \mathrm{~Hz}$ ausweisen, was genau der Frequenz des männlichen Balzrufes entspricht. In Nordamerika haben sich 2 Populationen vom Grillenfrosch sexuell isoliert, wobei die Männchen in New Jersey mit einer Frequenz von $3550 \mathrm{~Hz}$, jene in South Dakota mit $2900 \mathrm{~Hz}$ locken. Die Weibchen in New Jersey sind für die Lockrufe der Männchen aus South Dakota taub, weil ihre Hörneurone ein enges Empfindlichkeitsmaximum bei $3500 \mathrm{~Hz}$ haben ; das umgekehrte gilt für die Weibchen aus South Dakota (CAPRANICA and Rose, 1983).

Auch bei Grillen und Heuschrecken hat man im ZNS Detektoren für das digitale Weibchensignal gefunden. Für analoge Signale, wie Warnrufe oder Warndüfte steht hingegen ein Breitpassfilter zu Verfügung (Huber, 1983).

Wir müssen unserem Problem noch tiefer nachgehen : da der komplementäre Verständigungscode streng artspezifisch und damit angeboren ist, drängt sich die Frage auf : wie wird dieses Sender und Empfängersystem in den genetischen Code eingebaut - über genetische Koppelung, wonach Signal und Antwort im gleichen Chromosom codiert sind, oder nach dem Prinzip der Co-Evolution, das fordert, dass beide Systeme unabhängig einander zugeordnet wurden. Wie in vielen anderen Fällen, so hat auch hier die Natur beide Möglichkeiten verwirklicht.

Zunächst ein Beispiel für genetische Koppelung : Dr. Hoy von der Cornell- 
a
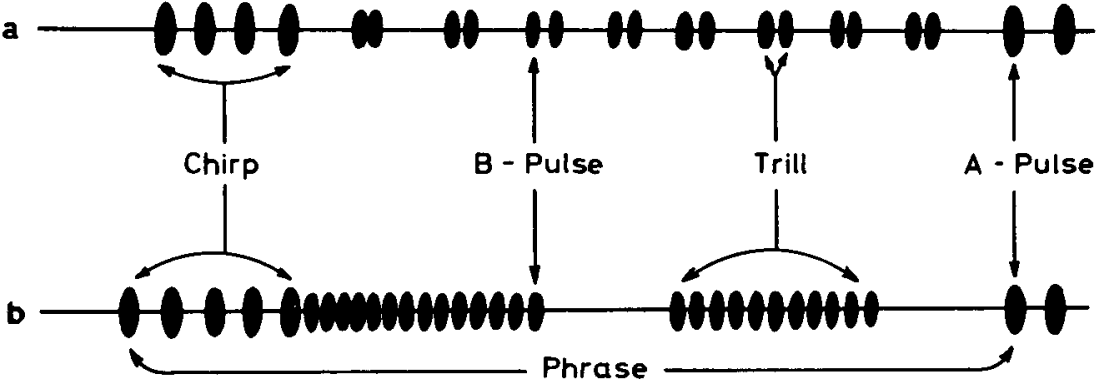

a Teleogryllus oceanicus

b Teleogryllus commodus

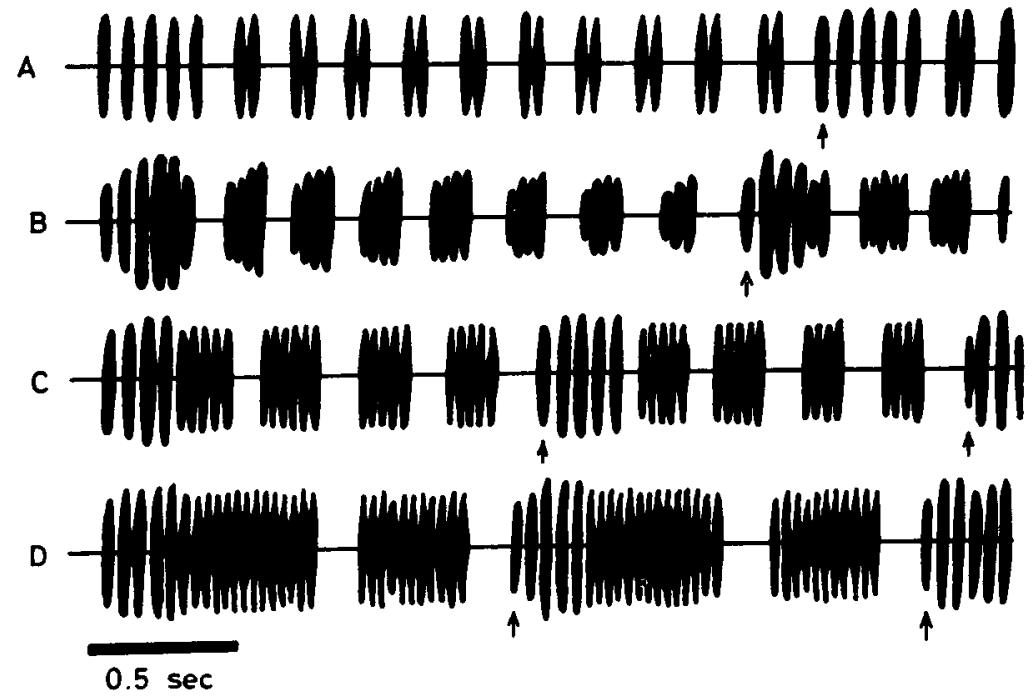

Aвв. 4. - Lautmuster des Werbegesanges zweier Grillenarten (Hoy, 1974).

a und A : Teleogrillus oceanicus.

b und D : Teleogrillus commodus.

B : Lautmuster der Tochtergeneration aus $T$. oceanicus - Weibchen $\times T$. commodus Männchen.

C : Lautmuster der Tochtergeneration aus $T$. commodus - Weibchen $\times T$. oceanicus - Männchen. 
University ist es gelungen zwei verschiedene Grillenarten (Teleogryllus oceanicus mit $T$. commodus) zu kreuzen. Man muss hierzu zunächst ein Weibchen von $T$. oceanicus, das man in einen Drahtkäfig gesperrt hat, durch den Werbegesang eines arteigenen Männchens motivieren. Auf dem Gipfel der Stimmung lässt man dann das Weibchen frei, vertauscht aber schnell das oceanicus Männchen mit dem Männchen von $T$. commodus, das vorher mit einem Weibchen seiner eigenen Art gebalzt hatte. Mit einigem Glück gelingt so eine Verbastardierung der zwei verschiedenen Arten. Die Männchen der F 1-Generation haben für ihren Werbegesang sowohl Anteile der Vater- wie der Muttergeneration übernommen, mit anderen Worten, die Vererbung des Senderapparates ist intermediär (Abb. 4). Die Frage ist nun, werden sich die Töchter in der F 1-Generation vom Gesang einer der Elterngenerationen oder von dem ihrer Brüder anlocken lassen. Das Ergebnis ist eindeutig : diese Töchter hören nicht auf ihren Vater, sondern interessieren sich nur für den Lockgesang ihrer Brüder. Daraus muss man zwingend folgern, dass Sender sowohl wie Empfänger ihren Signalcode in einem Chromosom in Form einer genetischen Koppelung eingelagert haben (Hoy, 1974).

Anders scheinen die Verhältnisse bei Heuschrecken (Helversen und HeLVERSEN, 1975) und beim Stichling zu liegen. Von unseren einheimischen Stichlingen ist bekannt, dass die Männchen mit ihrem roten Bauch die Weibchen zum Nest locken und zum Laichen veranlassen. Der einfache Schlüsselreiz : « rote Unterseite » löst die Balzhandlung aus. Überraschenderweise gibt es nun im Gebiet des Cehaly-Flusses im westlichen Nordamerika Stichlingsmännchen ohne roten Bauch. Sie haben ein pechschwarzes Prachtkleid. Ihr Balzverhalten ist etwas muihsamer und nicht so erfolgreich wie bei unseren Stichlingen; immerhin gelingt es ihnen über den Zickzacktanz das eine oder andere Weibchen anzulocken. In diesem Flussgebiet lebt ein kleiner schwarzer Raubfisch (Novumbra bubbsi), der sich mit Vorliebe von jungen Stichlingen ernährt. Die Stichlingspopulation dieses Flussbeckens war während der letzten Eiszeit isoliert und in dieser Periode der Isolation haben die Stichlingsmännchen durch mehrfache Mutation ein pechschwarzes Prachtkleid erworben. Sie sind damit nicht so auffällig und daher gegen die Angriffe des Räubers geschützt. Interessant wird die Angelegenheit erst, wenn man das Verhalten der Stichlingsweibchen studiert. Innerhalb des Gebietes der schwarzen Rasse lassen sie sich, wie eben geschildert, wenn auch zögernd, von den schwarzbauchigen Männchen verleiten in ihren Nestern zu laichen. Aber wenn man Weibchen der schwarzen Population im Aquarium die Wahl gibt zwischen einem schwarzbauchigen und einem rotbauchigen Männchen, so ziehen sie $5: 1$ ein rotbauchiges Männchen vor. Der Zeitraum von 8000 Jahren, seitdem die schwarzbauchige Rasse nunmehr existiert, hat also nicht ausgereicht, um das ursprünglich genetisch festgelegte Auslöseschema abzubauen. Wenn keine rotbauchigen Männchen da sind, weil sie der Raubfisch alle gefressen hat, dann laichen die Weibchen der Not gehorchend in den Nestern der schwarzbauchigen Männchen. Aber ihr «Traumbild»bleibt das rotbauchige Männchen. Ohne Zweifel liegt hier $\mathrm{Co}$ - 
Evolution vor, denn das Sendersignal war unabhängig durch Mutation abgeändert worden, während der Empfängercode nicht verändert wurde (MAYR, 1970).

Noch ein anderes Beispiel soll das Prinzip der Co-Evolution erläutern : die Fütterung der Silbermöwenküken wird durch zwei Schlüsselreize ausgelöst :

1) Das Elterntier gibt sich durch den roten Schnabelfleck zu erkennen.

2) Das Küken muss an diesem roten Schnabelfleck picken, um das Elterntier $\mathrm{zu}$ veranlassen das mitgebrachte Futter auszuwürgen.

TINBERGEN hat neben den vielen künstlichen Attrappen, die alle wirksam waren, sofern sie nur den roten Fleck an der richtigen Stelle hatten, einen überoptimalen Schlüsselreiz gefunden - einen roten Bleistift mit drei weissen Ringeln. Wie soll man solches deuten? Der Fachmann spricht hier von Präadaptation und meint damit, dass beim Küken, d.h. beim Empfänger, durch Mutation ein Signalmuster mit hohem Selektionswert der zu erwartenden komplimentären Mutation beim Elterntier, d.h. beim Sender, vorausgeeilt sei. Auf keinen Fall können hier Sender und Empfängercode im gleichen Chromosom nierdergelegt sein. Sie haben durch Co-Evolution unabhängig ihre Semantik erhalten.

Dies sind alles eindrucksvolle Beispiele, dass der Code des Sender- und des Empfängersystems voll und ganz angeboren ist. In den letzten Jahren sind bei Vögeln und Säugern auch Fälle bekannt geworden, dass Kommunikationssysteme adaptiv erlernt werden : MARLER an der Rockefeller University hat gefunden (mündl. Mitteilung), dass einige Singvögel in der Gegend von N. Millbrook verschiedene Dialekte singen - so wie auch unsere Buchfinken.

Da gibt es nun Männchen, die in dieser Hinsicht besonders begabt sind. Sie streunen viel herum, horchen dabei die Dialekte anderer Populationen ab und übernehmen sie für ihren eigenen Gesang. Das Interessante dabei ist, dass die Weibchen diese weitgereisten Vagabunden mit ihrem vielseitigen Dialekt in ihrer Partnerwahl bevorzugen, ein Prinzip, das sich in der Evolution bewährt hat : mit einem vielgereisten Partner hat man bessere Chancen neue ökologische Nischen für die Nachkommen zu finden.

THESE 3

These 3 soll ein andersartiges Problem behandeln, das z.Zt. unter Soziobiologen heftig diskutiert wird, besser gesagt heftig umstritten ist.

"Verstehen, Kommunikation, Verständigung setzen Mit-Teilung im wörtlichen Sinn voraus. Dies wiederum bedeutet, dass jeder Kommunikation altruistisches Verhalten zugrunde liegt ». Altruismus ist in diesem Zusamenhang so zu verstehen, 
dass Sender und Empfänger sich gegenseitig auf die Motivation des Partners und auf seine jeweilige Situation einstellen und im gegenseitigen Auffordern und Respondieren zu gemeinsamer Entscheidung und gemeinsamer Handlung kommen. Eigennutz, d.h. der reine Selbsterhaltungstrieb wird zugunsten der Arterhaltung zurückgestellt. Dieses Prinzip soll anhand der Arbeitsteilung im Bienenstaat erläutert werden.

$\mathrm{Da}$ ist zum ersten die erstaunliche Tatsache, dass der überwiegende Teil eines Bienenvolkes, nämlich die Arbeitsbienen, die etwa 60000-100000 Individuen ausmachen, auf eigene Nachkommen verzichten und sich ausschliesslich der Pflege ihrer Geschwister, d.h. der Töchter des einzigen fruchtbaren Wesens, der Königin, widmen. Innerhalb der Arbeitsbienen finden wir eine weitere Spezialisierung in Baubienen, Brutammen, Wächter und Trachtbienen. Um die Harmonie auf dem Arbeitsmarkt zu sichern, hat der Bienenstaat ein einzigartiges, im höchsten Masse altruistisches Kommunikationssystem ausgebaut, dessen Grundlage ein genetisch festgelegter Arbeitskalender bildet. Dadurch wird die Reihenfolge der einzelnen Tätigkeiten nach einem bestimmten Lebensalter festgelegt :

Jede Biene beginnt gleich nach dem Schlüpfen mit dem Reinigungsdienst, wozu vor allem das Sterilisieren der Brutzellen gehört; durch Austapezieren mit dem bakteriziden und fungiziden Mandibeldrüsensekret wird eine vorbildliche Hygiene für die Kinderstube der Bienen geschaffen. Nach etwa 3 Tagen geht die Biene zur Brutpflege über. Sie verfüttert Pollen und Honig an ältere Larven, während die jungen Larven das Sekret der Ammendrüsen sowie der Mandibeldrüse als Nahrung erhalten. Ab dem 10. Lebenstag etwa betätigt sich unsere Biene eine Woche lang als Bauarbeiterin. Schliesslich nimmt sie ihren Posten als Wächter vor dem Flugloch ein, bleibt da einige Tage und unvermittelt kommt sie dann mit Pollen oder Nektar beladen als Sammelbiene nach Hause. Bei dieser Tätigkeit wird sie bis zu ihrem Lebensende bleiben.

Zwei Tatbestände sind für eine erfolgreiche Verständigung auf dem Arbeitsmarkt der Bienen entscheidend :

1. Mit dem Wechsel von einer Tätigkeit zu einer anderen geht jeweils eine entsprechende physiologische Umstellung im Organismus vor sich : die Brutpflegerinnen haben hochaktive Pharynxdrüsen, die die «Ammenmilch» liefern. Die stark entwickelten Wachsdrüsen findet man nur bei den Baubienen; bei den Sammelbienen sind sowohl Pharynxdrüsen wie Wachsdrüsen vollständig zurückgebildet.

2. Das oben geschilderte Schema der Arbeitsteilung ist nicht so starr altersgebunden, wie dies zunächst scheinen mag. Ich habe mehrmals eine Biene mit der Stoppuhr Tag und Nacht von ihrem ersten Lebenstag an bis zu ihrem Tod verfolgt und festgestellt, dass sie zwar vorrangig den altersmässig zuständigen Arbeitsplatz einnimmt, dass sie aber auch anderweitig aushilft, wo « Not am Mann» ist. 
Erst diese adaptive Regulation garantiert die volle Harmonie der Tätigkeiten von 60000 bis 100000 Individuen in einem Bienenvolk. Sie wird besonders eindrucksvoll bei künstlichen Eingriffen vor Augen geführt : nimmt man einem Volk in einem sog. Umstellungsversuch alle Jungbienen, d.h. alle Brutbienen und Baubienen weg, dann unterziehen sich jene Bienen, die sich noch einigermassen jugendlich fühlen, einer Verjüngungskur : sie machen sich an die Pollentöpfe, regenerieren in wenigen Tagen durch die eiweissreiche Pollennahrung ihre Ammendrüsen und ihre Wachsdrüsen und repetieren ihr Lebensprogramm, von ihrer Jugendzeit angefangen, noch einmal. Es sei betont : nicht ein subjektives Bedürfnis, nur die sozialen Anforderungen lösen diese Verjüngungskur aus !

Umgekehrt, wenn man ein Volk durch Verstellen des Stockes « kahlfliegen 》 lässt, wobei es alle Sammelbienen verliert, dann wagen sich schon in den ersten Tagen nach der Umstellung jene Stockbienen, die mit dem 5. bis 6. Lebenstag ihre ersten Orientierungsflüge hinter sich gebracht haben und einige Geländeerfahrung besitzen, ins Freie und kommen alsbald mit Nektar und Pollen beladen in den Stock. Sie sind in ihren frühen Jugendtagen schon zu betagten Feldbienen geworden.

Solche elegante Anpassungsfähigkeit zugunsten der sozialen Bedürfnisse im Verband ist bewundernswert. Wir fragen : wer informiert die einzelnen Bienen jeweils, welche Tätigkeit sie zu jeder Stunde ausüben müssen, damit sich diese harmonisch den Bedürfnissen des Verbandes anpasst?

Es ist ein denkbar einfacher Mechanismus, der hier die Lösung bringt : die einzelne Biene erhält keine Anweisung von höherer Stelle; sie informiert sich selbständig über die jeweiligen Bedürfnisse im Verband. In ausgedehnten Patrouillengängen, die bei Stockbienen bis zu $60 \%$ der gesamten Tätigkeit ausmachen, inspizieren die Bienen immer wieder die verschiedenen Arbeitsplätze - Brutwaben, Bautraube u.a., und wo Hilfe nötig ist, springen sie ein - soweit ihr physiologischer Zustand dies irgendwie erlaubt (Lindauer, 1975).

Zwei Grundprinzipien garantieren Erfoig und Harmonie in diesem System :

1. Es liegt hier der Sonderfall einer unilateralen, einseitigen Information vor ; ein genetisch festgelegtes Verhaltensmuster und wohl auch individuelle Erfahrung geben erschöpfende Auskunft, was in jeder Situation zu tun ist.

2. Der Antrieb zu solchem sozialen Handeln setzt jedoch ein absolut selbstloses - altruistisches - Verhalten voraus. In der Wertung und kausalen Analyse sind die Soziobiologen in jüngster Zeit jedoch in heftige Kontroverse geraten :

Dürfen wir hier wirklich von einem echten altruistischen Verhalten sprechen ? Neuere Überlegungen meinen, es sei das «selfish gene », das « eigennützige Gen », das seinem Träger ein Verhaltensprogramm vorschreibt, das dem gesamten Verband, 
d.h. dem gemeinsamen Genpool zugute kommt. Da Altruismus erwiesenermassen umsomehr ausgeprägt ist, je höher der Verwandtschaftsgrad zwischen zwei Individuen ist, liege letztlich dem Dienst am Artgenossen Eigennutz für die eigenen Erbanlagen, die ja das Programm für das Verhalten enthalten, vor. «Inclusive Fitness » wird der Darwin'schen «Individual Fitness» gegenübergestellt. Die Situation im Bienenstaat wird dabei als Paradebeispiel genommen.

Gemäss den Mendel'schen Regeln gibt ja der Verwandtschaftsgrad genau an, mit welcher Wahrscheinlichkeit ein bestimmtes Gen beim Partner anzutreffen ist. Anders ausgedrückt : je näher verwandt zwei Individuen miteinander sind, desto mehr Gene haben sie gemeinsam. Wir bezeichnen zunächst den Verwandtschaftsgrad eines Individuums mit einem anderen als $\ll r$ und definieren diesen Verwandtschaftsgrad wie erwähnt als die Wahrscheinlichkeit mit der ein bestimmtes Gen auch in einem anderen Individuum anzutreffen ist.

Da können wir zu allererst feststellen, dass jedes Individuum mit sich selbst am engsten verwandt ist, denn die Zellen eines Organismus, Muskelzellen, Nervenzellen, Eizellen, haben zueinander den höchsten Verwandtschaftsgrad, sie haben den gleichen Chromosomensatz, also auch die gleichen Erbanlagen; ihr Verwandtschaftsgrad ist $\mathbf{r}=1$, das gleiche gilt auch für einige Zwillinge, auch hier ist $r=1$, denn sie haben einen identischen Genotyp. Zwischen Eltern und Kindern hingegen ist $r=1 / 2$, da ja die Nachkommen die Hälfte ihrer Erbanlagen vom Vater, die andere Hälfte von der Mutter haben.

Zwischen Geschwistern errechnet sich « $\mathrm{r} »$ wie folgt :

$$
r=1 / 2 \times 0,5+1 / 2 \times 0,5=1 / 2
$$

Hierzu folgende Erläuterung :

$1 / 2$ ergibt sich wie eben angedeutet dadurch, dass die Nachkommen eines Elterntieres je die Hälfte ihrer Erbanlagen vom Vater, bzw. von der Mutter haben, das gilt auch für ein Geschwister.

0,5 soll die Wahrscheinlichkeit ausdrücken, dass zwei Geschwister denselben Chromosomensatz von der Mutter bekommen haben, die ja für sich wieder zwei verschiedene Chromosomensätze zu vergeben hat, nämlich einen von ihrem eigenen Vater und einen von ihrer Mutter. Links vom Pluszeichen steht also die Wahrscheinlichkeit der Genverteilung für ein Geschwister, das das Erbgut von der Mutter, rechts vom Pluszeichen für ein Geschwister, das das Erbgut vom Vater hat. Insgesamt ergibt sich damit $r=1 / 2$. Dies alles gilt natürlich nur, wenn die Geschwister den gleichen Vater und die gleiche Mutter haben.

Für Halbgeschwister gilt $r=1 / 4$.

$$
\mathrm{r}=1 / 2 \times 0,5+1 / 2 \times 0=1 / 4
$$


Besonders interessant sind nun die Verhältnisse bei Bienen und Ameisen, an denen Hamilton ursprünglich seine Theorie entwickelt hat. Wir finden da die Besonderheit, dass aus befruchteten Eiern Weibchen, aus unbefruchteten Männchen entstehen. Die Weibchen haben also zwei Chromosomensätze (diploid), die Männchen haben nur einen, sie sind haploid. Die Männchen haben demgemäss keinen Vater und können auch keine Söhne haben; sie haben nur eine Mutter, einen Grossvater und eine Grossmutter mütterlicherseits. Der Verwandtschaftsgrad der weiblichen Arbeitsbienen untereinander errechnet sich daher wie folgt :

$$
\mathbf{r}=1 / 2 \times 0,5+1 / 2 \times 1=3 / 4
$$

\section{Zur Erklärung :}

Links vom Pluszeichen steht die Genkombination, die von der Mutter, rechts jene, die vom Vater stammt. $1 / 2$ heisst, dass ein Chromosomensatz entweder vom Vater oder von der Mutter stammt, 0,5 ist die Wahrscheinlichkeit, dass das gleiche Gen unter Geschwistern geteilt wird. Nach dem Pluszeichen bedeutet wiederum 1/2, dass ein Chromosomensatz vom Vater oder von der Mutter stammen kann. 1 zeigt an, dass zwei Schwestern mit Sicherheit den gleichen Chromosomensatz vom Vater haben. Dies ist also der Verwandtschaftsgrad Arbeiterin zu Arbeiterin.

Da die Königin nur einen ihrer beiden Chromosomensätze an jede Tochter weitergeben kann, ist « $r » z w i s c h e n$ Mutter und Tochter $1 / 2$. Wir können aus dieser Gleichung die schwerwiegende Folgerung ziehen : im Bienenvolk sind Schwestern untereinander näher verwandt als Mutter und Tochter.

Der Verwandtschaftsgrad zwischen Brüdern, also zwischen Drohnen, errechnet sich wie folgt :

$$
r=1 \times 0,5=1 / 2
$$

Die Männchen bekommen ja ihr gesamtes Erbgut von der Mutter. Verwandtschaftsgrad Bruder zu Schwester, also Drohn zu Arbeiterin :

$$
r=\frac{1+0}{2}=1 / 2
$$

d.h. ein Bruder hat entweder alles oder nichts mit seiner Schwester gemein.

Für den Verwandtschaftsgrad zwischen Arbeiterin und Drohn, also Schwester zu Bruder, ist $\mathrm{r}=1 / 2 \times 0,5=1 / 2$.

Das ergibt eine merkwürdige Asymmetrie im gegenseitigen Verwandtschaftsverhältnis : ein Bruder ist mit seiner Schwester näher verwandt, als die Schwester mit ihm - sofern Verwandtschaftsgrad wie hier definiert, die Wahrscheinlichkeit kennzeichnet, dass ein Gen im Partner wiederzufinden ist. Denn die Schwester kann mit einem Bruder nur jene Hälfte ihres Erbgutes (1/2) gemeinsam haben, die sie von der Mutter bekam; vom Vater hat ja der Bruder keinen Anteil. 
Für den Verwandtschaftsgrad Tochter zu Vater ergibt sich $r=1 / 2$, für Vater zu Tochter jedoch $r=1$. Eine Ungleichheit des reziproken Verwandtschaftsgrades besteht also auch zwischen Vater und Tochter; die Tochter hat die Hälfte ihres Erbgutes vom Vater $r=1 / 2$, der Vater hingegen findet mit Sicherheit sein ganzes Erbgut in der Tochter, daher $\mathrm{r}=1$.

Wenn wir nun die sozialen Hilfeleistungen der einzelnen Kasten im Bienenstaat mit ihrem Verwandtschaftsgrad in Vergleich setzen, ergibt sich eine Schlussfolgerung, die in der Tat die Theorie des «selfish gene» bestätigt : die Arbeiterrinnen pflegen die Larven ihrer Mutter, d.h. die Nachkommen der Königin ; das sind aber die eigenen Schwestern, mit denen sie, wie wir betont haben, näher verwandt sind, als sie es mit ihren eigenen Nachkommen wären, auf die sie verzichten; ohne Zweifel : sie verschaffen damit ihrem eigenen Erbgut den grössten Dienst. Man kann auch so sagen : die Königin wird in der Brutpflege von den unfruchtbaren Arbeiterinnen entlastet; damit helfen diese ihrer Mutter weitere Schwestern in grosser Zahl zu erzeugen, die wiederum 3/4 ihres eigenen Erbgutes tragen. Hätten sie selber Nachkommen, dann könnten sie ihre Fürsorge nur der Hälfte ihres Erbgutes zugute kommen lassen.

Das «selfish gene » wirkt in dem Sinne, dass es neben der Darwin'schen «Individual Fitness 》 durch «Kin Selection», d.h. durch Sippenselektion die «Inclusive Fitness》 im engsten Verwandtschaftskreis einer Population fördert (Hamilton, 1970, 1972 ; Wilson, 1975).

Wie so oft in den komplexen biologischen Systemen stossen diese theoretischen Überlegungen in der Praxis auf Abweichnugen - vielleicht sind es nur Ausnahmen oder nachträgliche Anpassungserscheinungen. Um bei den Bienen zu bleiben :

1. Neuere Untersuchungen haben erwiesen, dass eine Bienenkönigin bei ihrem Hochzeitsflug in der Regel nicht nur einmal, sondern mehrmals hintereinander begattet wird, natürlich jedesmal von einem anderen Drohn. Das Verwandtschaftsverhältnis unter den Arbeitsbienen, die also verschiedene Väter haben, wird dadurch sehr heterogen.

2. Jeder Imker weiss, dass man - unter entsprechenden Vorsichtsmassnahmen - ohne weiteres in einem Bienenvolk die Brutwaben mit denen aus einem anderen Volk austauschen kann, dass man Brutammen einem fremden Volk zusetzen kann, dass man sogar die Königin beliebig ersetzen kann - die sozialen Instinkte der Brutpflege und der harmonischen Arbeitsteilung bleiben dabei voll erhalten - auch wenn sie jetzt einem fremden Erbgut gelten.

Die recht primitive Verständigungsform bei der Arbeitsteilung hat Nachteile :

1. Im gesamten System steckt eine hohe statistische Unsicherheit, denn das Auffinden eines neuen Arbeitsplatzes durch ständiges Patrouillieren ist stark zufallsbedingt. 
2. Dieses Patrouillieren bedeutet erheblichen Zeitverzug, das gesamte Kommunikationssystem arbeitet recht träge.

Für die Nahrungssuche, wo es darum geht, die aufblühende Tracht möglichst schnell einzutragen, ehe die Blüten ihre Kelche wieder schliessen und ehe die Konkurrenz das Futter wegnimmt, wäre diese Art der Kommunikation völlig unbrauchbar.

Hier muss gezielte, eindeutige und schnelle Information übermittelt werden. Die Kommunikation muss weiterhin Massenwerbung sein, d.h. sie muss simultan eine grosse Anzahl von Stockgenossen erfassen und zu einer spezifischen Handlung auffordern. Dies geschieht durch den bekannten Bienentanz.

In der Tat liegt hier ein Verständigungssystem vor, das an detaillierten, gezielten, schnell wirkenden Informationen im Tierreich unübertroffen ist.

\section{THESE 4}

«Der Bienentanz nimmt eine Sonderstellung unter allen tierischen Kommunikationssystemen ein » er benutzt :

1. symbolische Semanteme;

2. die Mitteilung erfolgt unabhängig vom Ort und von der Zeit des Geschehens ;

3. die gleichen Semanteme können für verschiedene Situationen angewendet werden. >

Zunächst ganz kurz der Informationsgehalt des Tanzes, wie ihn uns Karl v. Frisch $(1923,1946,1965)$ beschreibt ; eine erfolgreiche Sammlerin meldet ihren Nestgenossen :

1. Dass sie eine lohnende Futterquelle entdeckt hat. Sie verbindet diese Meldung mit der Aufforderung an die Kolleginnen, bei der Ausbeute der Tracht zu helfen.

2. Sie teilt die Art der Blüten mit, die besucht wurden; dies geschieht mit Hilfe von Duftproben, die sie mit dem Nektar und in ihrem Haarkleid aus den Blüten mitgenommen hat. Während die Nachtänzerinnen sie mit ihren Fühlern berühren und den Nektar abnehmen, nehmen sie auch diesen Duft auf.

3. Die Sammlerin gibt durch die Lebhaftigkeit des Tanzes darüber Auskunft, wie das Futter beschaffen ist und ob sich seine Ausbeute lohnt ; sie alarmiert nur, wenn Nektar und Pollen reichlich zur Verfügung stehen und der Zuckergehalt des Nektars hoch ist. Dabei lösen im Frühjahr, zur Zeit der Haupttracht, nur Nektarquellen mit hohem Zuckergehalt (60-80\%) Tänze aus. Im Herbst jedoch, wenn 
kaum noch Tracht zu finden ist, tanzen die Sammelbienen immer noch mit der gleichen Lebhaftigkeit, wenn man den Nektar um das Zehnfache verdünnt.

4. Die grösste Überraschung erlebte Karl v. FRISCH aber, als er Hinweise erhielt, dass neben der Qualität, Rentabilität und Blütenart auch die Lage der Futterstelle mitgeteilt wird. Nahe Futterziele kündet ein Rundtanz an. Wenn das Futtertischchen über $100 \mathrm{~m}$ hinaus wandert, geht der Rundtanz in einen Schwänzeltanz über. In ihm ist eine überraschend genaue Entfernungs- und Richtungsanweisung enthalten :

Die Entfernung zum Ziel wird durch eine veränderliche Frequenz des Schwänzellaufes angezeigt. Richten wir unsere Futterstelle stufenweise bei verschiedenen Entfernungen von 100-10000 m ein und vergleichen die Frequenz der Schwänzelläufe miteinander, so erhalten wir eine Beziehung zu der Entfernung. Schon auf den ersten Blick lässt sich feststellen, dass diese Frequenz bei nahen Futterzielen viel höher ist als bei ferner gelegenen. So zählen wir bei $200 \mathrm{~m}$ Entfernung etwa 8 Durchläufe je $1 / 4$ Min., bei $2000 \mathrm{~m}$ Entfernung nur noch 3 und bei $10000 \mathrm{~m}$ Entfernung 1 1/2 Durchläufe.

Wenn die Tänzerinnen so genau die Entfernung weisen können, müssen sie diese natürlich vorher selbst ebenso genau vermessen haben.

Sie richten sich hierbei nicht etwa, wie man lange Zeit annahm, nach der Flugzeit, sondern nach dem Energieaufwand, den sie zur Bewältigung der jeweiligen Entfernung aufbringen müssen. Versuche an einem Berghang haben dies bestätigt :

Eine Bienenschar wurde veranlasst, vom Stock aus bergauf $\mathrm{zu}$ fliegen, eine andere bergab. Die Bergauf-Sammlerinnen meldeten eine längere Entfernung als die Bergab-Sammlerinnen, obwohl beide Futtertischchen vom Stock gleich weit entfernt waren. Desgleichen melden die Tänzerinnen bei Gegenwind eine grössere, bei Rückenwind eine kleinere Entfernung. Die Flugzeit ändert sich unter den gegebenen Bedingungen nur unwesentlich; aber der Energieaufwand steht in direkter Beziehung zur Entfernungsweisung. Es bleibt aber immer noch zu klären, wie die Biene den Energieverbrauch so genau messen kann. Mein Mitarbeiter, V. NeEsE, konnte auch diese Frage lösen :

Jede Biene nimmt für ihren Flug zum Futterziel eine bestimmte Menge Honig als Reiseproviant mit. Dieser Proviant wird direkt als Energiereserve durch die Darmwand resorbiert. Dadurch nimmt die Spannung in der Honigmagenwand ab ; hochempfindliche Spannungsmesser registrieren diesen Spannungsabfall, der proportional zur zurückgelegten Flugstrecke ist.

Die Angabe über die Entfernung einer Futterquelle würde aber einem Bienenvolk wenig nützen, wenn die Tänzerinnen nicht auch zugleich die Richtung bekannt gäben. Um festzustellen wie das geschieht, schicken wir unsere Sammelschar an ein 
Futtertischchen $600 \mathrm{~m}$ im Süden und beobachten ihre Tänze über den ganzen Tag. Dabei stellen wir fest, dass sich die Richtung der Schwänzelläufe vom Morgen bis zum Abend entgegen dem Uhrzeigersinn ändert, und zwar genau entsprechend der Azimutwinkelgeschwindigkeit der Sonne (Azimut $=$ Projektion der jeweiligen Sonnenstellung auf den Horizontkreis der Erde). Am Morgen weist der Schwänzellauf $90^{\circ}$ nach rechts, schwenkt dann bis Mittag senkrecht nach oben ein und erreicht schließlich bis $6.00 \mathrm{Uhr}$ abends die Linie $90^{\circ}$ links. Man kann sagen, dass sämtliche Tanzrichtungen der Lotrechten nach links und rechts zugeordnet werden, je nachdem, ob die Flugbahn links oder rechts zur Sonne verläuft. Schwänzellauf nach oben heisst also : das Ziel liegt in Richtung zur Sonne. Schwänzellauf nach unten bedeutet : «Ihr müsst von der Sonne wegfliegen, damit ihr zum Ziel kommt ». Schwänzellauf $80^{\circ}$ links von der Lotrechten zeigt ein Ziel an, das $80^{\circ}$ links von der Sonne liegt. Schwänzellauf $90^{\circ}$ rechts von der Lotrechten weist zu einem Ziel, das $90^{\circ}$ rechts von der Sonne liegt.

Man ist versucht, den Bienentanz als Verständigungsform einer echten Sprache gegenüberzustellen, was nicht heisst, dass wir der menschlichen Sprache ihre Einzigartigkeit als Kommunikationsform nicht voll und ganz zuerkennen ; aber einige charakteristische Elemente einer echten Sprache finden wir auch im Bienen$\operatorname{tanz}$ : HocketT (1960) und SEbeok $(1965,1972)$ nennen als wesentliche Kennzeichen einer Sprache : Broadcast, Semanticity by using symbols, Displacement, Productivity, Interchangeability, Duality.

Ansätze für alle genannten Kennzeichen finden wir auch im Bienentanz :

a) Die Tänzerin übermittelt erfolgreich Nachrichten : sie meldet ihren Stockgenossinnen, dass sie eine ergiebige Futterquelle entdeckt hat; dadurch werden beschäftigungslose Arbeitsbienen veranlasst, auszufliegen und nach dieser Futterquelle zu suchen (Broadcast, Productivity).

b) Diese Nachrichtenübermittlung ist nicht an den Ort und an die Zeit des Geschehens gebunden. So hat eine Sammelbiene beispielsweise in $2,5 \mathrm{~km}$ Entfernung vom Stock Nektar gesammelt; sie meldet den Fund aber nicht unmittelbar an Ort und Stelle, sondern erst nach ihrer Rückkehr im Stock (Displacement).

c) Die Tänzerin benutzt Symbolzeichen, um die Lage und Ergiebigkeit der Fundstelle anzuzeigen. «Symbol » lässt sich nach dem «Dictionary of the English Language» definieren als : «Something used for or regarded as respresenting else $\gg$.

Rhythmische Schwänzelbewegungen beziehen sich in ihrer zeitlichen Abfolge genau auf die Entfernung des Zieles; der Winkel zwischen Flugbahn und Sonne, transponiert ins Schwerefeld, zeigt die Richtung zum Ziel an. Die Lebhaftigkeit des Tanzes zeigt die Güte des Futters an (Semanticity).

d) Die in den Tanzbewegungen verwendeten «Zeichen » können für ver- 
schiedene Gegebenheiten ausgewechselt werden - für Nektar-, Pollen- und Wassersammeln — aber auch für neuentdeckte Niststätten (LiNDaUER, 1954) (Interchangeability, Versatility).

Der wesentliche Unterschied zwischen der Bienensprache und einer echten Sprache besteht demnach darin, dass die Bienensprache ein starres Codesystem ist, das nicht durch Austausch und Abwandlung seiner Symbole schwierige Aufgaben der Darstellung erfüllen kann. Ausserdem sind die Grundbestandteile der Bienensprache angeboren, ihre Semanteme können nicht neu zusammengestellt, nicht erlernt und durch Überlieferung weitergegeben werden. Die Bienensprache kennt keine Syntax. Schliesslich kann die Biene - wie jedes andere Tier - dem Artgenossen keine Fragen stelllen. Eine direkte Antwort wird vom Partner nicht gegeben - während doch gerade das wandelbare Gespräch ein wesentlicher Bestandteil der echten Sprache ist, in dem durch Austausch und Abwandlung seiner Symbole auch schwierige Aufgaben der Darstellung erfüllt werden.

Gelegentlich wurden wir gefragt, ob Bienen auch lügen können. Heute ist meine Antwort darauf ein klares Nein. Aber jahrelang waren wir da in einem Dilemma : wir mussten feststellen, dass die Tänzerinnen gelegentlich die Richtung zum Ziel falsch anzeigten. Was uns erregte, war, dass sie nicht statistisch Fehler machten, sondern gemeinsam nach einem System eine sog. "Missweisung » zeigten ; beispielsweise liefen ihre Tanzwinkel am Vormittag der Sonnenwanderung voraus, am Nachmittag hinkten sie hinterher. Wenn wir den Stock über Nacht um $90^{\circ}$ drehten, war am nächsten Tag die Tendenz der Missweisung umgekehrt. Heute wissen wir, dass da keine Lüge dahintersteckt, es handelt sich auch nicht um eine Missweisung - unsere Interpretation, gegründet auf unsere menschliche Sinneswahrnehmung war zu naiv : nicht nur die Sonne und die Schwerkraft, auch das Erdmagnetfeld wird bei der Richtungsweisung als stabilisierender Parameter mit einbezogen. Hier hat sich uns eine Welt aufgetan, die unseren Sinnen nicht zugänglich ist : Bienen können das Erdmagnetfeld wahrnehmen und in seiner Intensität und seinen richtungsbezogenen Komponenten ( $x, y-z-K o m p o n e n t e)$, die für jeden geographischen Ort, sowie für jede Tages- und Jahreszeit spezifisch sind, für ihre Orientierung in Raum und Zeit nutzen (Lindauer und Martin, 1968).

Unsere Befunde an Bienen haben weltweit Anregung gegeben, auch bei anderen Tiergruppen nach biomagnetischen Wirkungen zu suchen. Heute wissen wir, dass das Erdmagnetfeld von vielen Tieren zur Orientierung benutzt wird, dass es auf der anderen Seite auch vielerlei Einfluss auf physiologische Prozesse im Organismus hat. Kein Wunder, dass sich auch die Medizin zunehmend für das neue Forschungsgebiet «Biomagnetismus » interessiert (MARTIN und LiNDaUER, 1973). 

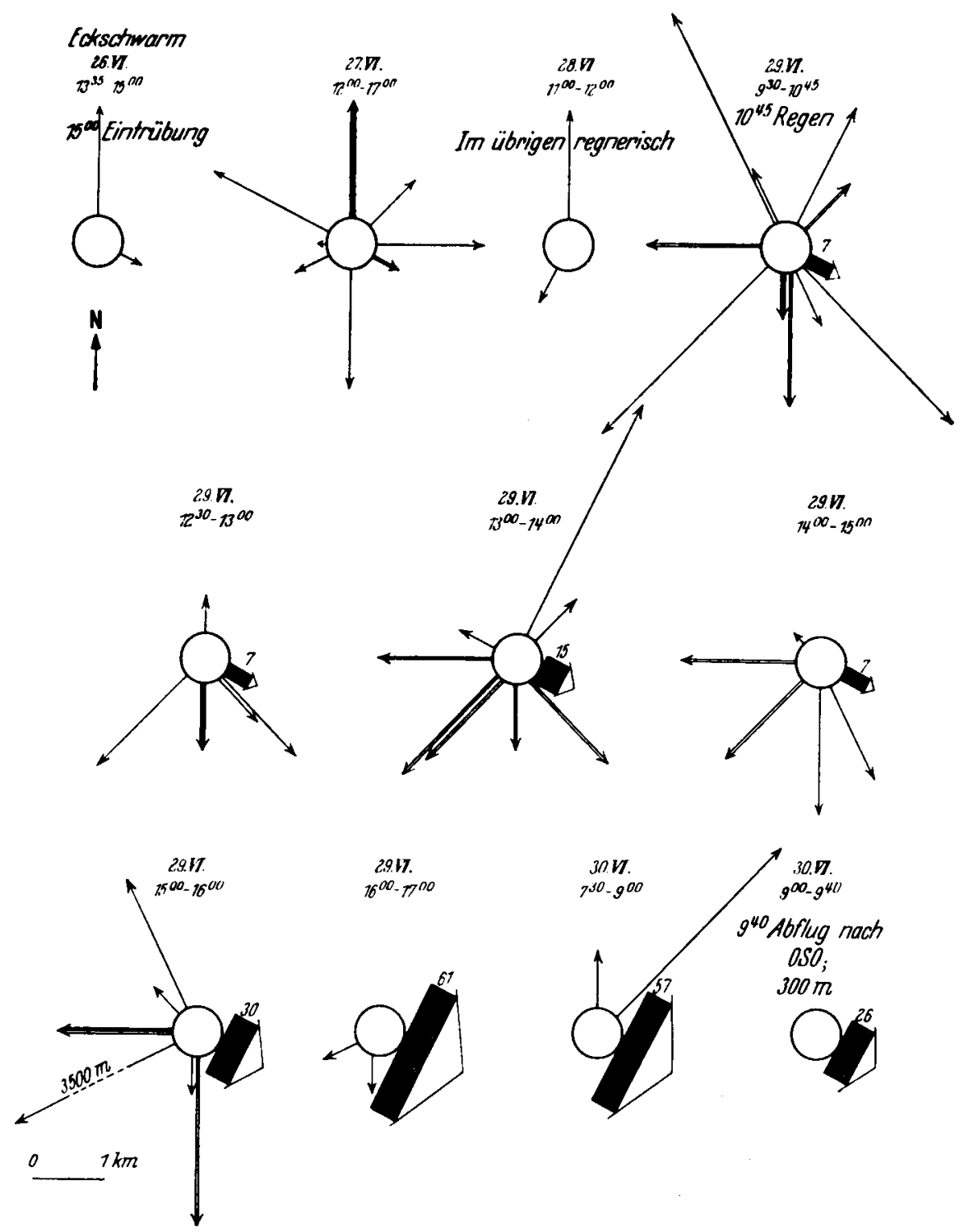

Aвв. 5. - Von den Schwarmspurbienen wurden durch Tänze auf der Traube verschiedene Nistplätze angekïndigt.

Jeder Pfeil gibt maßstabgerecht Richtung und Entfernung der potentiellen Niststätte an. Es erfolgt eine Einigung auf den Nistplatz Ost-Süd-Ost $300 \mathrm{~m}$. 


\section{WOHNUNGSSUCHE}

Die Verständigung durch Rund- und Schwänzeltanz dient nicht nur dem Nahrungserwerb, sondern auch der Wohnungssuche. Wenn ein Bienenschwarm aus dem Mutterstock auszieht, sammelt er sich zunächst als Traube in der Nähe des Stockes. Wenn der Imker nicht gleich zur Stelle ist, ziehen bald einige Dutzend Spurbienen aus, um die Umgebung nach günstigen Niststätten abzusuchen. Sie schwärmen zunächst in alle Himmelsrichtungen; diejenigen unter ihnen, die eine passende Höhle in einem alten Baum, in einer Ruine oder in einem leeren Fuchsbau gefunden haben, fliegen zur Traube zurück und zeigen ihren Stockgenossinnen die Fundstelle durch einen Schwänzeltanz an, der in gleicher Weise die Richtung und Entfernung des Zieles angibt, wie der Tanz der Sammelbienen. Die alarmierten Schwarmbienen fliegen dann ab und prüfen die gemeldete Niststätte; sofern sie auch ihnen als neuer Wohnsitz geeignet erscheint, werben sie weitere "Prüfer * an. Ich habe bei solchen Versuchen die tanzenden Spurbienen mit Farbtupfen gekennzeichnet und auf einem Messtischblatt die angekündigten Niststätten eingezeichnet; in einigen Fällen ist es uns gelungen, den Nistplatz schon aufzufinden, ehe der Schwarm eingezogen war. Es gibt wohl keinen besseren Beweis dafür, dass wir wirklich die «Sprache» der Bienen verstehen und richtig deuten können.

Bei diesen Untersuchungen mussten wir aber die überraschende Feststellung machen, dass die Quartiermacher durch ihre Tänze nicht nur einen, sondern mehrere Nistplätze zu gleicher Zeit anmelden. In einem Fall gingen auf der Traube 24 verschiedene Meldungen ein. Wie werden sich nun die zwanzigtausend oder dreissigtausend Bienen einer Schwarmtraube einig darüber, welchen der angebotenen Nistplätze sie beziehen sollen ? Der Schwarm kann sich ja nicht teilen - er muss geschlossen mit seiner Königin in eine der angebotenen Wohnungen einziehen. Diese Einigung kommt auch tatsächlich zustande. Die Tänze werden immer einheitlicher ; schliesslich deuten sie nur noch auf einen bestimmten Nistplatz hin (Abb. 5). Nun löst sich die Schwarmtraube auf und zieht geschlossen in das neue Heim um. Es kann aber auch sein, dass sich das Interesse der Tänzerinnen gleichzeitig auf zwei Nistplätze konzentriert. In solchen Fällen muss gleichfalls zuerst eine Einigung erfolgen, indem eine der beiden Gruppen nachgibt und ihre Werbung einstellt. Wie erfolgt diese Einigung? Es hat sich herausgestellt, dass die Entscheidung stets den Spurbienen überlassen bleibt. Sie treffen wirklich eine gute Wahl : unfehlbar entscheiden sie sich für den besten der aufgefundenen Nistplätze. Damit sie sich aber untereinander verständigen können, wo der beste Platz liegt, teilt jede Spurbiene nicht nur die Lage, sondern auch die Güte der entdeckten Wohnung mit. Handelt es sich um erstangige Nistplätze, so wird äusserst lebhaft und ausdauernd, ja stundenlang durchgetanzt, während ein minderwertiges Quartier nur zaghaft durch einen kurzen, matten Tanz von wenigen Minuten Dauer 
angemeldet wird. Das hat zur Folge, dass von Anfang an mehr Neulinge für den erstrangigen Nistplatz geworben werden. Die auf diese Weise verständigten Bienen besichtigen die verschiedenen Angebote und werben nach ihrer Rückkehr weitere Neulinge für die jeweils beste Wohnung an. Entscheidend ist aber, dass auch diejenigen Spurbienen, die zunächst mittelmässige Nistplätze angezeigt hatten, sich für die Meldungen ihrer Kolleginnen interessieren, die anderen Plätze prüfen und durch Vergleich von sich aus für den besten unter ihnen werben. Mit anderen Worten : die Schwarmspurbienen lassen sich umstimmen. Hier unterscheiden sie sich grundsätzlich von den Sammelbienen, die ja starr so lange bei ihrer Sammelgruppe bleiben, bis der Nektar versiegt ; erst dann sieht man, dass sie sich für die Tänze einer anderen Gruppe interessieren (Lindauer, 1955).

\section{SCHLUSS}

Es sei eine Schlussbemerkung erlaubt : Die Versuchung ist gross, Gesetzmässigkeiten und Organisationsprinzipien tierischer Sozialverbände, die sich seit mehr als 100 Millionen Jahren bewährt haben, als Empfehlung an die HumanSoziologen weiterzugeben. Ich würde mich dem persönlich widersetzen : der Homo sapiens und die menschliche Gesellschaft verdanken ihre Sonderstellung im Reich der lebenden Organismen nicht nur dem Umstand, dass der Mensch mit Vernunft, Einsicht und logischem Denken begabt ist ; entscheidend für diese Sonderstellung ist, dass er sich von den Fesseln der genetischen Verhaltensprogramme gelöst hat, er hat die Fähigkeit zu freier Willensentscheidung und drittens : ihm steht über die echte Sprache die Möglichkeit offen, wichtige kulturelle Errungenschaften als Tradition weiterzugeben.

Die Frage freilich bleibt, ob mit dem kulturellen und wissenschaftlichen Fortschritt auch die ethisch begründete Verantwortung des Einzelnen für die Gesamtheit in unserer Zeit Schritt halten kann.

Eingegangen im April 1984.

\section{LITERATURVERZEICHNIS}

CaPranica R.R. and Rose G., 1983. - Frequency and Temporal Processing in the Auditory System of Anurans. In : Neuroethology and Behavioral Physiology, Hubert F., MarkL H. ed., Springer-Verlag Heidelberg, New York, Tokyo, 137-152.

Frisch K. von, 1923. - Über die «Sprache» der Bienen, eine tierpsychologische Untersuchung. Zool. Jb. (Physiol.), 40, 1-186.

Frisch K. von, 1946. - Die Tänze der Bienen. Österr. Zool. Z., 1, 1-48.

FriSCH K. von, 1965. - Tanzsprache und Orientiertung der Bienen. Springer-Verlag, Berlin, Heidelberg, New York, $578 \mathrm{p}$. 
Hamilton W.D., 1970. - Selfish and spiteful behavior in a evolutionary model. Nature, London, 228 (5277), 1218-1220.

Hamilton W.D., 1972. - Altruism and related phenomena, mainly in social insects. Annu. Rev. Ecol. Syst., 3, 193-232.

Helversen O.H. v., Helversen D., 1975. - Verhaltensgenetische Untersuchungen am akustischen Kommunikationssystem der Feldheuschrecken (Orthoptera, Acrididae). 1. Der Gesang von Artbastarden zwischen Chorthippus biguttulus u. Ch. mollis. J. comp. Physiol., 104, 173-299. II. Das Lautschema von Artbastarden zwischen Chortippus biguttulus und Ch. mollis. J. comp. Physiol, 104, 301-323.

HocketT C.F., 1960. - Logical considerations in the study of animal communication. In : W.E. LanYon and W.N. Tavolga eds. (q.v.), Animal sounds and Communication, pp. 392-430.

Hoy R.R., 1974. - Genetic Control of Acoustic Behavior in Crickets. Am. Zool., 14.

Huber F., 1983. - Neural Correlates of Orthopteran and Cicada Phonotaxis. In : Hurer F., MarkL H. ed., Neuroethology and Behavioral Physiology Springer-Verlag, Heidelberg, New York, Tokyo, 108-135.

Lindauer M., 1955. - Schwarmbienen auf Wohnungssuche. Z. vgl. Physiol., 37, 263-324.

Lindauer M., 1975. - Verständigumg im Bienenstaat. Stuttgart, Gustav Fischer Verlag, 163 p.

L.indauer M. und Martin H., 1968. - Die Schwereorientierung der Bienen unter dem Einfluss des Erdmagnetfeldes. Z. vgl. Physiol., 60, 219-243.

Martin H. und Lindauer M., 1973. - Orientierung im Erdmagnetfeld. Fortschr. Zool, 21 (2/3), 211-228.

MaYr E., 1970. - Evolution und Verhalten. Verh. Dtsch. Zool. Ges. Köln, Gustav-Fischer Verlag, Stuttgart.

Priesner E., 1973. - Artspezifität und Funktion einiger Insektenpheromone. Fortschr. Zool., Bd. 22, Heft 1 «Orientierung der Tiere im Raum», Gustav Fischer-Verlag, Stuttgart.

Sевеок, 1965. - Animal communication. Science, 147, 1006-1014.

Wilson E.O., 1975. - Sociobiology : The New Synthesis. Cambridge (Mass.), The Belknap Press of Harvard Univ. Press. 